\title{
Competition Among Providers in Loss Networks
}

\author{
Patrick Maillé • Bruno Tuffin
}

Received: date / Accepted: date

\begin{abstract}
Communication networks are becoming ubiquitous and more and more competitive among revenue-maximizing providers, operating on potentially different technologies. In this paper, we propose to analyze the competition of providers playing with access prices and fighting for customers. Considering a slotted-time model, the part of demand exceeding capacity is lost and has to be resent. We consider an access price for submitted packets, thus inducing a congestion pricing through losses. Customers therefore choose the provider with the cheapest average price per correctly transmitted unit of traffic.

The model is a two-level game, the lower level for the distribution of customers among providers, and the upper level for the competition on prices among providers, taking into account what the subsequent repartition at the lower level will be. We prove that the upper level has a unique Nash equilibrium, for which the user repartition among different available providers is also unique, and efficient in the sense of social welfare. Moreover, even when adding a higher level game on capacity disclosure with a possibility of lying for providers, providers are better off being truthful, and the unique Nash equilibrium is thus unchanged.
\end{abstract}

Keywords Competition · Game theory · Wireless Networks · Pricing · Resource allocation

Mathematics Subject Classification (2000) $90 \mathrm{~B} 10 \cdot 91 \mathrm{~A} 80$

P. Maillé

Institut Telecom/Telecom Bretagne, 2 rue de la Châtaigneraie CS17607, 35576 Cesson-Sévigné Cedex, FRANCE.

Tel.: +33-299127028

Fax: +33-299127030

E-mail: patrick.maille@telecom-bretagne.eu

B. Tuffin

INRIA Rennes Bretagne Atlantique, Campus Universitaire de Beaulieu, 35042 Rennes Cedex, FRANCE.

Tel.: +33-299847494

Fax: +33-299847171

E-mail: bruno.tuffin@inria.fr 


\section{Introduction}

Telecommunication networks are now managed by commercial service providers trying to attract customers in order to maximize their revenue. A typical example is the Internet: The network was indeed initially just a connection of academic and cooperative sites, but it has now moved to a much broader entity, whose access for customers is enabled by selfish and competitive providers. Furthermore, instead of having a network per application, all applications (telephony, email, web browsing, video, games...) can now be carried out using any technology, being the ADSL network, FTTx, 3G wireless networks, WiFi or WiMAX (or LTE), with heterogeneous quality of service (QoS) capabilities. This convergence leads to a complex system which requires to be analyzed from an economical point of view, taking into account the technological specificities.

\subsection{Contribution}

We propose in this paper to study a competition game among providers with heterogeneous and non-overlapping capacities (or spectrum if dealing with wireless). Those providers are modeled by loss networks, such that if demand at a provider exceeds capacity, demand in excess is lost and has to be resent. Congestion pricing is applied by charging for sent traffic instead of successfully received one. More precisely, the more traffic is observed, the more likely packets are to be lost and then resubmitted (and paid again for). As a result, the total price charged per successfully received packet, named here perceived price, is an increasing function of demand. Customers are assumed infinitesimal, i.e., the strategy of a single individual does not have any impact on others: only a grouped action of a bunch of customers can affect congestion levels, and thus perceived prices. Therefore, when they act selfishly, their global repartition will obey the so-called Wardrop's principle [22], initially introduced in the (equivalent) transportation domain: only providers with the cheapest perceived price obtain some demand. We show that whatever the access price at providers, there exists such a user equilibrium situation, and that the (common) perceived price at all providers with positive demand is unique. Knowing how customers will distribute themselves for any combination of prices, providers try to maximize their revenue by playing with their prices. We therefore end up with a two-level Stackelberg game [10], where the providers are the leaders, using by backward induction the anticipated decision (the repartition) of customers to determine their strategy. We show that there exists a unique Nash equilibrium for the pricing game, and we characterize it explicitly. A Nash equilibrium is a price profile such that no provider can unilaterally improve its revenue. We show that this non-cooperative case actually and surprisingly leads to the same configuration than the cooperative case, when all actors, i.e., providers and customers, jointly try to maximize the sum of their utilities -also known as social welfare-.

This paper is related to [16], where the same pricing tools were applied, but users were assumed to be sensitive to their total submitted traffic, not for received one. As a consequence, lost packets were somewhat considered as satisfactory because they were not resubmitted. We consider here the more realistic situation where traffic that counts is the successfully transmitted one. Even if the results look similar to those in [16], that new model requires a reformulation of the problem and a complete rewriting of all proofs. 
1.2 Related work

The general framework of the paper is that of non-cooperative game theory [10]. In telecommunications, game theory has been used a lot in the last decade to model the behavior of distributed algorithms, with potentially selfish actors (see for example [4] and references therein).

We deal here more specifically with telecommunications pricing, a topic of active research $[6,7,18-20]$. Remark however that most of the studies are dealing with a monopoly, whereas we consider here an oligopoly. Oligopolies have been extensively studied in other areas than telecommunications [21], but telecommunication networks have specificities (e.g., congestion effects on QoS) that are not encompassed by most models. Moreover, competition is a reality in the current telecommunication world, and needs to be taken into account, since it can lead to significantly different results than monopoly situations [11]. For other competition models, with different assumptions and atomic users, the reader can look at, among others, [8,9,13-15,17].

The case of users' distribution following Wardrop's principle has been considered in [5], where price competition among producers is studied without congestion effects on the user side, but with a negative externality on the supply side through some production costs. Our model also has some demand-related costs (that we interpret as management costs), but we consider that their level is low with respect to revenues, as can be expected in wireless networks where most costs come from infrastructure and are independent of demand. We moreover introduce a particular form of negative externality on the user level, that is typical for limited capacity networks with losses. Other references $[2,3,12]$ apply Wardrop's principle on the user level to study competition. In all those papers the externality is the expected delay, not the loss probability like here.

As described in the previous subsection, the present paper is related to one of our previous works [16], but we now include the fact that retransmissions are taken into account in the demand level. It is actually a more relevant and key new assumption, that leads to completely different proofs.

\subsection{Organization of the paper}

This paper is organized as follows. Section 2 presents the general model. Section 3 defines the socially-optimal situation, i.e., the cooperative situation with providers and customers jointly maximizing social welfare. In Section 4, we describe and characterize how customers distribute themselves, following Wardrop's principle, for any fixed profile of provider prices. Using that user equilibrium, Section 5 shows that there exists a unique equilibrium for the price competition among providers, and that the corresponding outcome is actually socially optimal. Section 6 studies the potentiel interest for providers to lie about their real capacities in a competitive environment and then to artificially increase congestion for a potential larger revenue due to resent packets. Finally, Section 7 summarizes the contributions and presents directions for future research. 


\section{General model}

We consider a set $\mathcal{I}:=\{1, \ldots, I\}$ of $I \geq 2$ providers in competition at an access point. Time is slotted and each provider $i(i \in \mathcal{I})$ can serve $C_{i}>0$ packets (or units, seen as a continuous number) per slot. If demand exceeds capacity at a given provider, demand in excess is lost. Lost packets are assumed to be chosen uniformly over the set of submitted ones. If $d_{i}$ is the total demand at provider $i$, the number of served packets is actually $\min \left(d_{i}, C_{i}\right)$, meaning that packets are actually served with probability $\min \left(C_{i} / d_{i}, 1\right)$. Users are assumed to be charged a price $p_{i}$ for each submitted packet instead of each served one. This induces a congestion pricing to yield incentives to limit demand, the negative externality of congestion being expressed in terms of losses experienced by users. The total income of provider $i$ is $d_{i} p_{i}$ and the total service "rate" is $d_{i} \min \left(C_{i} / d_{i}, 1\right)$. Then the average perceived price per served traffic unit at provider $i$ is therefore

$$
\underline{p}_{i}=p_{i} / \min \left(C_{i} / d_{i}, 1\right)=p_{i} \max \left(d_{i} / C_{i}, 1\right) .
$$

Charging on sent packets instead of successfully transmitted ones may seem unrealistic. However, that mechanism can be seen as a volume-based pricing scheme, with a congestion-dependent charge. Somewhat equivalently, it can also be seen as a consequence of the more frequently used time-based charging with a fixed price per time unit. Indeed, when congestion occurs on a network $i$ and packets are lost, having to send them again multiplies the total transfer time (and thus the price paid) by the mean number of transmissions per packet $\max \left(1, d_{i} / C_{i}\right)$.

We assume that total user demand is a function $D(\cdot)$ of the perceived price $\underline{p}$, and that $D$ is continuous, derivable, and strictly decreasing with $\underline{p}$ on its support $\left[0, p_{\max }\right.$ ) (with possibly $p_{\max }=+\infty$ ), and that $\lim _{p \rightarrow+\infty} D(p)=0$. We moreover assume that $D(0)>\sum_{i \in \mathcal{I}} C_{i}$, i.e., that there is some congestion: the total resource available is not sufficient to satisfy the maximum demand level. Finally, we assume that $D(0)<+\infty$ : if the access were free, then the total demand would be finite. Remark that this last assumption can be easily met, by considering the sending capacity limits of user machines.

We also define the function $v: q \mapsto \inf \{p: D(p) \leq q\}$ (with the convention $\inf \emptyset=0$ ), that we call the marginal valuation function at the $q$-th unit of demand. From our assumptions on $D, v(q)$ is finite for all $q \geq 0$. From an economic point of view, $v(q)$ represents the maximum price per traffic unit at which the $q$ traffic units could be sold.

We finally define $V(q)$, the overall valuation, as the sum of the marginal valuations of the $q$ units of users with largest willingness-to-pay, i.e.,

$$
V(q):=\int_{x=0}^{q} v(x) d x .
$$

The economic interpretation of $V(q)$ is the total value of the first $q$ served units of traffic, for users who are willing to pay the most for the service. Those marginal and overall valuation functions will be useful to characterize the socially-optimal situation and the distribution $\mathbf{d}:=\left(d_{1}, \ldots, d_{I}\right)$ of customers among providers, obtained from a given price profile.

The goal of each provider $i$ is, by playing on its unit price $p_{i}$, to maximize its net benefit

$$
R_{i}\left(p_{1}, \ldots p_{I}\right):=p_{i} d_{i}-\ell_{i}\left(d_{i}\right)
$$


where $p_{i} d_{i}$ is the money earned directly from demand, and $\ell_{i}\left(d_{i}\right)$ represents the cost for provider $i$ of managing a demand level $d_{i}$. We assume that for all $i, \ell_{i}$ is nondecreasing.

Most of our results are valid under the following assumption preventing provider management cost functions from being too steep. Remark that this assumption seems reasonable, since management costs are in general very small with respect to infrastructure costs (that are independent on current demand, and thus not considered here), and with respect to incomes from customers.

Assumption A The management cost function $\ell_{i}$ of every provider $i \in \mathcal{I}$ is Lipschitzcontinuous on $\left[0, C_{i}\right]$ with a Lipschitz constant $\kappa_{i}$ smaller than the global marginal valuation of the sum of all provider capacities. In other terms,

$$
\forall i \in \mathcal{I}, \forall x, y \leq C_{i}, \quad\left|\ell_{i}(x)-\ell_{i}(y)\right| \leq \kappa_{i}|x-y|,
$$

with $\kappa_{i} \leq v\left(\sum_{j \in \mathcal{I}} C_{j}\right)$.

Remark 1 Remark that Assumption A is satisfied for example if the functions $\left(\ell_{i}\right)_{i \in \mathcal{I}}$ are derivable and convex, and such that

$$
\forall i \in \mathcal{I}, \quad \ell_{i}^{\prime}\left(C_{i}\right) \leq p^{*}
$$

where $\ell_{i}^{\prime}$ is the derivative of $\ell_{i}$, and $p^{*}=v\left(\sum_{j \in \mathcal{I}} C_{j}\right)$.

For some results, we will need a stricter assumption, that includes an elasticity condition on demand:

Assumption B In addition to Assumption A, we assume that for unit prices larger than $p^{*}:=v\left(\sum_{j \in \mathcal{I}} C_{j}\right)$, the demand function $D$ is sufficiently elastic:

$$
y \geq p^{*} \quad \Rightarrow \quad \frac{-y D^{\prime}(y)}{D(y)} \geq \frac{1}{1-\kappa / y},
$$

where $\kappa_{i}$ is the Lipschitz constant for the cost $\ell_{i}$ on $\left[0, C_{i}\right], \kappa:=\max _{i \in \mathcal{I}} \kappa_{i}$, and $D^{\prime}$ is the derivative of the demand function $D$.

Remark 2 When management costs are negligible (i.e., $\kappa_{i}=0$ for all $i \in \mathcal{I}$ ), then Assumption B consists in demand elasticity being larger than 1, an assumption often made in economics to describe situations where demand is highly sensitive to prices.

\section{Socially optimal situation}

Following usual vocabulary from economics, we define Social welfare as the sum of utilities of all actors in the game -here, users and providers-. The total user utility is the overall user valuation minus the total price paid, while the total provider utility (revenue) is the total price paid minus the total managing cost. Therefore, prices do not directly appear in the expression of social welfare.

Proposition 1 For a demand configuration $\mathbf{d}:=\left(d_{1}, \ldots, d_{I}\right)$, social welfare is expressed by the quantity

$$
S W(\mathbf{d}):=\frac{\sum_{i \in \mathcal{I}} \min \left(d_{i}, C_{i}\right)}{\sum_{i \in \mathcal{I}} d_{i}} V\left(\sum_{i \in \mathcal{I}} d_{i}\right)-\sum_{i \in \mathcal{I}} \ell_{i}\left(d_{i}\right) .
$$


Proof The first term in SW is the total valuation for the service experienced by users. Indeed, $V(x)$ is the total user valuation, if the $x$ users with largest willingness-to-pay are served. For a given demand configuration, the total quantity served is $\sum_{i \in \mathcal{I}} \min \left(d_{i}, C_{i}\right)$. Moreover, when demand exceeds capacity, then not all demand is served: among total demand $\sum_{i \in \mathcal{I}} d_{i}$, only $\sum_{i \in \mathcal{I}} \min \left(d_{i}, C_{i}\right)$ are served, the others getting no service and thus having a zero valuation. Since we assume that losses occur regardlessly of user willingness-to-pay, the actual (per traffic unit) utility of a user having (per traffic unit) willingness-to-pay $v$ is its willingness-to-pay times the probability to be served, i.e.,

$$
\frac{\sum_{i \in \mathcal{I}} \min \left(d_{i}, C_{i}\right)}{\sum_{i \in \mathcal{I}} d_{i}} v .
$$

User are assumed infinitesimal, therefore the total user valuation equals

$$
\int_{u=0}^{\sum_{i \in \mathcal{I}} d_{i}} \frac{\sum_{i \in \mathcal{I}} \min \left(d_{i}, C_{i}\right)}{\sum_{i \in \mathcal{I}} d_{i}} v(u) d u,
$$

the first term in (2). The second term in (2) is simply the total managing cost for the demand $\mathbf{d}$.

In our next result, we characterize the most efficient demand vector $\mathbf{d}$, in the sense of social welfare. A priori, that demand configuration may not correspond to users selfishly selecting their provider.

Proposition 2 Under Assumption A, social welfare is maximized when $d_{i}=C_{i}$ for each provider $i$.

Proof We consider any demand vector $\mathbf{d}$, and we prove that truncating the demand $d_{i}$ to the capacity of each provider $i \in \mathcal{I}$ can only increase social welfare. Defining a new demand vector $\mathbf{d}^{\mathrm{n}}=\left(\min \left(d_{i}, C_{i}\right)\right)_{i \in \mathcal{I}}$, we have

$$
\begin{aligned}
\operatorname{SW}\left(\mathbf{d}^{\mathrm{n}}\right)= & V\left(\sum_{i \in \mathcal{I}} \min \left(d_{i}, C_{i}\right)\right)-\sum_{i \in \mathcal{I}} \ell_{i}\left(\min \left(d_{i}, C_{i}\right)\right) \\
\geq & \frac{\sum_{i \in \mathcal{I}} \min \left(d_{i}, C_{i}\right)}{\sum_{i \in \mathcal{I}} d_{i}} V\left(\sum_{i \in \mathcal{I}} d_{i}\right) \\
& -\sum_{i \in \mathcal{I}} \ell_{i}\left(\min \left(d_{i}, C_{i}\right)\right) \\
\geq & \operatorname{SW}(\mathbf{d}),
\end{aligned}
$$

where the second line comes from $V$ being a concave function with $V(0)=0$, which implies that $\alpha V(x) \leq V(\alpha x)$ for any $x \geq 0$ and $0 \leq \alpha \leq 1$. The third line simply comes from the nondecreasingness of cost functions $\left(\ell_{i}\right)_{i \in \mathcal{I}}$.

As a result, we can look for an optimal demand profile $\mathbf{d}_{\text {opt }}$ in the compact convex set $\mathcal{C}:=\prod_{i \in \mathcal{I}}\left[0, C_{i}\right]$. The objective function being continuous, such an optimal profile always exists.

Now compare such an optimal demand $\mathbf{d}_{\text {opt }}$ to the profile $\mathbf{d}_{C}:=\left(C_{1}, \ldots, C_{I}\right)$. Since both profiles are in the set $\mathcal{C}$, we have 
$\mathrm{SW}\left(\mathbf{d}_{\mathrm{opt}}\right)-\mathrm{SW}\left(\mathbf{d}_{C}\right)$

$$
\begin{aligned}
& =V\left(\sum_{i \in \mathcal{I}} d_{i}\right)-V\left(\sum_{i \in \mathcal{I}} C_{i}\right)+\sum_{i \in \mathcal{I}}\left(\ell_{i}\left(C_{i}\right)-\ell_{i}\left(d_{i}\right)\right) \\
& \leq v\left(\sum_{i \in \mathcal{I}} C_{i}\right) \sum_{i \in \mathcal{I}}\left(d_{i}-C_{i}\right)+\sum_{i \in \mathcal{I}}\left(\ell_{i}\left(C_{i}\right)-\ell_{i}\left(d_{i}\right)\right) \\
& \leq \sum_{i \in \mathcal{I}}\left(v\left(\sum_{i \in \mathcal{I}} C_{i}\right)-\kappa_{i}\right)\left(d_{i}-C_{i}\right) \leq 0 .
\end{aligned}
$$

where we used the concavity of $V$ on the second line, and Assumption A on the last line. This concludes the proof: the demand vector $\mathbf{d}_{C}$ performs as least as well as any other demand vector in terms of social welfare.

\section{Wardrop equilibrium for users}

Let us investigate the necessary and sufficient conditions for a demand vector $\mathbf{d}$ to be a user equilibrium following Wardrop's principle [22]. That principle states that users always choose the cheapest options, so that for a stable situation, all users who have the same set of available options end up paying the exact same price. It was first introduced to model driver route choices in transportation, but can easily be applied to our problem, yielding:

1. Providers getting some demand have the same perceived price, which is the cheapest one. This can be written as

$$
d_{i}>0 \Rightarrow p_{i} \max \left(1, d_{i} / C_{i}\right)=\min _{j \in \mathcal{I}} p_{j} \max \left(1, d_{j} / C_{j}\right)
$$

Indeed, if a provider has a positive demand and a larger perceived price than a competitor, then part of its customers would churn to the cheapest.

2. The total amount of data that users want to successfully transmit depends on the perceived price per successful transmission. This writes

$$
\sum_{i \in \mathcal{I}} \min \left(d_{i}, C_{i}\right)=D(\underline{p})
$$

where

$$
\underline{p}:=\min _{j \in \mathcal{I}} p_{j} \max \left(1, d_{j} / C_{j}\right)
$$

i.e., the lowest perceived price among all providers. The left-hand side of (4) is the total rate of successful transmission, that takes into account the capacity limitations of each provider's access network.

This allows to formally define the user equilibrium.

Definition 1 For given capacity $\mathbf{C}:=\left(C_{1}, \ldots, C_{I}\right)$ and price $\mathbf{p}=\left(p_{1}, \ldots, p_{I}\right)$ configurations, a user equilibrium is a demand configuration $\mathbf{d}=\left(d_{1}, \ldots, d_{I}\right)$ such that for all $i, j \in \mathcal{I}$, 


$$
\left\{\begin{array}{l}
d_{i}>0 \Rightarrow p_{i} \max \left(1, d_{i} / C_{i}\right) \leq p_{j} \max \left(1, d_{j} / C_{j}\right), \\
\sum_{k \in \mathcal{I}} \min \left(d_{k}, C_{k}\right)=D\left(\min _{i \in \mathcal{I}} p_{i} \max \left(1, d_{i} / C_{i}\right)\right) .
\end{array}\right.
$$

Condition (5) re-expresses (3), the fact that all providers with positive demand have the same perceived unit price, otherwise part of the demand will have interest in changing providers. Condition (6) is a formulation equivalent to (4). The assumption that received data is the quantity of interest is by using $r_{k}=\min \left(d_{k}, C_{k}\right)$ in (5) instead of $d_{k}$ if we were using the amount of sent data.

Remark that we can equivalently write a user equilibrium as a vector $\mathbf{d}$ such that $(\mathbf{d}, \underline{p})$ is a solution of the system

$$
(S)\left\{\begin{array}{rlrl}
\left(p_{i} \max \left(1, d_{i} / C_{i}\right)-\underline{p}\right) d_{i}=0, & \forall i \in \mathcal{I} \\
p_{i} \max \left(1, d_{i} / C_{i}\right)-\underline{p} \geq 0, & \forall i \in \mathcal{I} \\
\sum_{i \in \mathcal{I}} \min \left(d_{i}, C_{i}\right)-D(\underline{p}) & =0, & \\
d_{i} \geq 0, & \forall i \in \mathcal{I} \\
\underline{p} & \geq 0 . &
\end{array}\right.
$$

In the system $(S), p$ stands for the common value of the perceived price at all providers that get demand.

The following proposition characterizes the user equilibria corresponding to fixed capacities and prices.

Proposition 3 For any capacity and price configuration where prices are strictly positive, there exist a (possibly not unique) user equilibrium demand configuration. Moreover, at a user equilibrium $\mathbf{d}$, the common perceived unit price $\underline{p}$ of providers $i$ with $d_{i}>0$ is unique and equals

$$
\begin{gathered}
\underline{p}=\min \left\{p: D(p) \leq \sum_{i \in \mathcal{I}} f_{i}(p)\right\} \\
\text { where } f_{i}(p):=C_{i} 1_{\left\{p \geq p_{i}\right\}}
\end{gathered}
$$

with $l_{X}$ the indicator function, of value 1 if condition $X$ is verified, and 0 otherwise.

Remark that we have a min in (12), since $D$ is continuously nonincreasing and $f_{i}$ is right-continuous and nondecreasing for all $i \in \mathcal{I}$.

Proof We follow the same steps as those taken in [1] to establish the existence of a solution for the system $(S)$. But the results of [1] do not directly apply, due to the distinction between demand flow $\mathbf{d}$ and successful flow $\mathbf{r}$, thus we adapt the proof. We first show that $(S)$ is equivalent to the nonlinear complementarity problem described 
by the system

$$
\left(S^{\prime}\right)\left\{\begin{aligned}
\left(p_{i} \max \left(1, d_{i} / C_{i}\right)-\underline{p}\right) d_{i} & =0 \forall i \in \mathcal{I}, \\
p_{i} \max \left(1, d_{i} / C_{i}\right)-\underline{p} & \geq 0 \forall i \in \mathcal{I}, \\
\left(\sum_{i \in \mathcal{I}} \min \left(d_{i}, C_{i}\right)-D(\underline{p})\right) \underline{p} & =0, \\
\sum_{i \in \mathcal{I}} \min \left(d_{i}, C_{i}\right)-D(\underline{p}) & \geq 0, \\
d_{i} & \geq 0 \forall i \in \mathcal{I}, \\
\underline{p} & \geq 0 .
\end{aligned}\right.
$$

A solution of $(S)$ is obviously a solution of $\left(S^{\prime}\right)$. Now consider a solution $(\mathbf{d}, p)$ of $S^{\prime}$ : if it is not a solution of $(S)$, then we necessarily have $\underline{p}=0$ and $\sum_{i \in \mathcal{I}} \min \left(d_{i}, C_{i}\right)>D(\underline{p})$. This last inequality means that there exists $i \in \mathcal{I}$ with $d_{i}>0$, which implies from (14) that $p=p_{i} \max \left(1, d_{i} / C_{i}\right)>0$, a contradiction.

Therefore the set of Wardrop equilibria corresponds to the set of solutions of $\left(S^{\prime}\right)$, which we now show is non-empty. First define a constant $K_{1}<+\infty$ satisfying

$$
\left\{\begin{array}{l}
K_{1}>\max _{i \in \mathcal{I}} \frac{C_{i} v\left(C_{i}\right)}{p_{i}} \\
K_{1}>\max _{i \in \mathcal{I}} C_{i}
\end{array}\right.
$$

which exists under our assumptions on $D$, and a constant $K_{2}<+\infty$ such that

$$
K_{2}>\max _{i \in \mathcal{I}}\left\{K_{1} p_{i} / C_{i}\right\}
$$

Remark that we then have

$$
\forall i \in \mathcal{I}, \quad D\left(p_{i} K_{1} / C_{i}\right)<C_{i} .
$$

We define the function $\Phi: \mathbb{R}^{|\mathcal{I}|+1} \rightarrow \mathbb{R}^{|\mathcal{I}|+1}$ by

$$
\Phi(\mathbf{d}, \underline{p})=\left(\Phi_{1}(\mathbf{d}, \underline{p}), \ldots, \Phi_{|\mathcal{I}|+1}(\mathbf{d}, \underline{p})\right)
$$

with $\Phi_{i}(\mathbf{d}, \underline{p})=$

$$
\left\{\begin{array}{l}
\min \left(K_{1},\left[d_{i}-p_{i} \max \left(1, d_{i} / C_{i}\right)+\underline{p}\right]^{+}\right) \text {if } i \leq|\mathcal{I}|, \\
\min \left(K_{2},\left[\underline{p}+D(\underline{p})-\sum_{j \in \mathcal{I}} \min \left(d_{j}, C_{j}\right)\right]^{+}\right) \text {for } i=|\mathcal{I}|+1
\end{array}\right.
$$

where $[x]^{+}$stands for the positive part of $x$.

Since $\Phi$ is a continuous function that maps the cube $\left[0, K_{1}\right]^{|\mathcal{I}|} \times\left[0, K_{2}\right]$ onto itself, from Brouwer's fixed point theorem it admits a fixed point $(\hat{\mathbf{d}}, \hat{p})$. We now prove that this point is a solution of $\left(S^{\prime}\right)$, i.e., it is a Wardrop equilibrium.

- Assume that $\hat{d}_{i}=K_{1}$ for some $i \in \mathcal{I}$, then from (22) we have $\underline{p} \geq p_{i} \max \left(1, K_{1} / C_{i}\right)>$ 0 . Thus $(21)$ implies that $D(\underline{\hat{p}})<C_{i}=\min \left(\hat{d}_{i}, C_{i}\right) \leq \sum_{j \in \mathcal{I}} \min \left(\hat{d}_{j}, C_{j}\right)$. Consequently, from $(23)$ and $(\hat{\mathbf{d}}, \underline{\hat{p}})$ being a fixed point of $\Phi$, we have $\underline{\hat{p}}=0$, which is a contradiction. 
- Likewise, if $\underline{\hat{p}}=K_{2}$ then from (20),

$$
\underline{\hat{p}}>p_{i} K_{1} / C_{i} \geq p_{i} \max \left(1, \hat{d}_{i} / C_{i}\right) \quad \forall i \in \mathcal{I},
$$

where the second inequality comes from $K_{1}>C_{i}$ and $\hat{d}_{i} \leq K_{1}$. This implies from (22) that $\hat{d}_{i}=K_{1}, \forall i \in \mathcal{I}$, which cannot happen as proved just before.

As a result, $(\hat{\mathbf{d}}, \hat{p})$ is a solution of the system

$$
\left\{\begin{array}{l}
\hat{d}_{i}=\left[\hat{d}_{i}-p_{i} \max \left(1, \hat{d}_{i} / C_{i}\right)+\underline{\hat{p}}\right]^{+} \quad \forall i \in \mathcal{I} \\
\underline{\hat{p}}=\left[\underline{\hat{p}}+D(\underline{\hat{p}})-\sum_{i \in \mathcal{I}} \min \left(\hat{d}_{i}, C_{i}\right)\right]^{+},
\end{array}\right.
$$

which is exactly equivalent to the system $\left(S^{\prime}\right)$. Thus we have proved the existence of a Wardrop equilibrium.

Now we consider a Wardrop equilibrium, and characterize the minimum perceived price $p$. From Condition (8),

$$
p_{i}<\underline{p} \Rightarrow d_{i}>C_{i}
$$

while from (7) we get

$$
\begin{aligned}
& p_{i}>\underline{p} \Rightarrow d_{i}=0, \\
& p_{i}=\underline{p} \Rightarrow d_{i} \leq C_{i} .
\end{aligned}
$$

Using Inequality (24), then (9) and finally (25), we get

$$
\sum_{i \in \mathcal{I}} \mathbb{1}_{\left\{p_{i}<\underline{p}\right\}} C_{i} \leq D(\underline{p})=\sum_{i \in \mathcal{I}} \min \left(d_{i}, C_{i}\right) \leq \sum_{i \in \mathcal{I}} \mathbb{1}_{\left\{p_{i} \leq \underline{p}\right\}} C_{i}
$$

which gives (12).

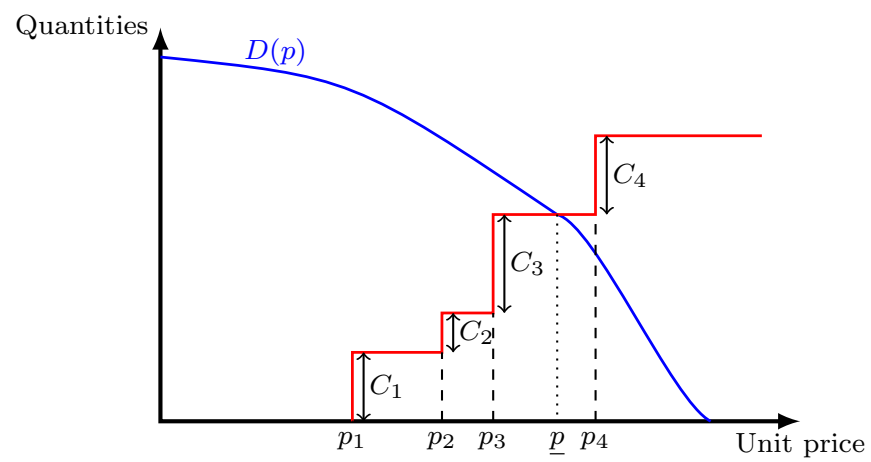

Fig. 1 Wardrop equilibrium for four providers and a given price configuration: the common perceived price at each provider with positive demand (i.e., providers $1,2,3$ ) is $\underline{p}$. Here the intersection occurs on an horizontal part of the stairstep curve.

Remark 3 Figures 1 and 2 display the two possible configurations for determining the Wardrop equilibrium perceived price $\underline{p}$. Either the stairstep curve summing up the capacities at the charged prices crosses the demand function on a horizontal part, or it happens on a vertical part. In any case, the existence and uniqueness of $p$ are ensured (because one curve is increasing while the other is strictly decreasing), as shown in Proposition 3. 


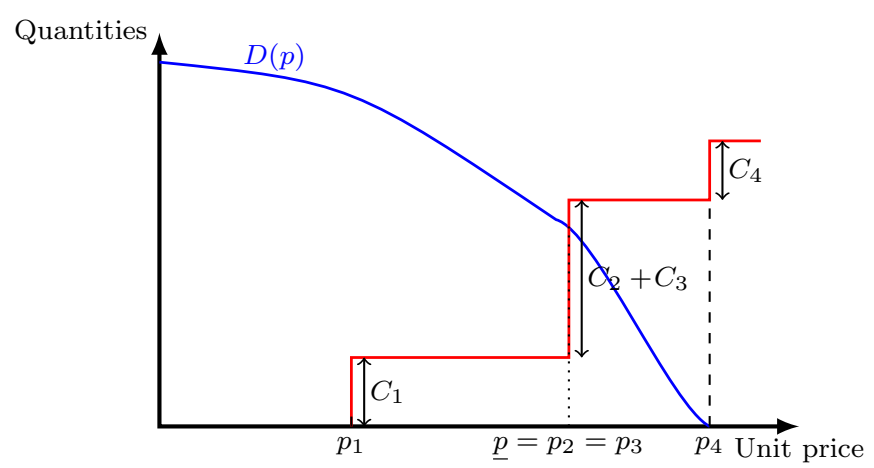

Fig. 2 Wardrop equilibrium for four providers and a given price configuration: the common perceived price at each provider with positive demand (i.e., providers $1,2,3$ ) is $\underline{p}$. Here the intersection occurs on a vertical part of the stairstep curve.

Remark 4 Total demand served is therefore $D(p)$. For all providers with price $p_{i} \neq p$, demand $d_{i}$ is then $d_{i}=\mathbb{1}_{\left\{p_{i}<\bar{p}\right\}} C_{i} \underline{p} / p_{i}$. All providers such that $p_{i}=\underline{p}$ (if any) share the remaining demand $D(\underline{p})-\sum_{j: p_{j}<\underline{p}} d_{j}$, all possible sharing with $0 \leq d_{i} \leq C_{i}$ providing a Wardrop equilibrium. That situation is illustrated in Figure 2. In that sense, there is not always uniqueness for the Wardrop equilibrium, and the corresponding revenues for each provider are not necessarily unique. Note nonetheless that the resulting total revenue is always the same. Moreover, we will see in the following that when providers are at a Nash equilibrium of the pricing game, then the corresponding user Wardrop equilibrium is unique.

\section{Price competition among providers}

In this paper, we consider that providers setting their prices is the upper stage of a two-level game, where the lower stage corresponds to users reacting according to the Wardrop equilibrium described in Definition 1. We assume that providers are aware of their advantage of playing first, i.e., they anticipate and take into account users' reaction when determining their price. That common knowledge complicates the competition among providers, and is the purpose of the analysis in this section.

Our main result is a complete characterization of the Nash equilibrium of the pricing game, taking benefit from the above corresponding characterization of the Wardrop equilibrium.

Proposition 4 Under Assumption B, there exists a Nash equilibrium of the price war among providers, given by

$$
\forall i \in \mathcal{I}, \quad\left\{\begin{array}{l}
p_{i}=p^{*} \\
d_{i}=C_{i}
\end{array}\right.
$$

where $p^{*}=v\left(\sum_{j \in \mathcal{I}} C_{j}\right)$, that is

$$
\sum_{i \in \mathcal{I}} C_{i}=D\left(p^{*}\right)
$$


Moreover, if cost functions $\left(\ell_{i}\right)_{i \in \mathcal{I}}$ are strictly increasing, then there is no other Nash equilibrium.

In words, the proposition means that at equilibrium, all providers set the same price, such that demand equals the total capacity of the system.

Proof The proof can be decomposed into two steps:

1. We first show that if cost functions are strictly increasing, only the point such that $d_{i}=C_{i}$ and $p_{i}=p^{*} \forall i$, with $p^{*}=v\left(\sum_{i \in \mathcal{I}} C_{i}\right)$ can be a Nash equilibrium;

2. then we prove that that point is indeed a Nash equilibrium. Remark that we do not need the strict increasingness of cost functions for that part.

Step 1: Uniqueness of the Nash equilibrium.

Assume that there exists a price configuration $\mathbf{p}$ that is a Nash equilibrium of the pricing game, and decompose the set of providers $\mathcal{I}$ into three disjoint subsets: $\mathcal{I}=\mathcal{I}_{s} \cup \mathcal{I}_{0} \cup \mathcal{I}_{u}$, where

$$
\begin{aligned}
& \mathcal{I}_{s}:=\left\{i \in \mathcal{I}: d_{i}>C_{i}\right\}, \\
& \mathcal{I}_{0}:=\left\{i \in \mathcal{I}: d_{i}=C_{i}\right\}, \\
& \mathcal{I}_{u}:=\left\{i \in \mathcal{I}: d_{i}<C_{i}\right\} .
\end{aligned}
$$

We will show that $\mathcal{I}_{s}$ and $\mathcal{I}_{u}$ are empty sets, which then implies from (7) and (9) that the price configuration is $\mathbf{p}=\left(p^{*}, \ldots, p^{*}\right)$.

We first prove that $\mathcal{I}_{s}=\emptyset$. Assume it is not the case, and consider $i_{s} \in \mathcal{I}_{s}$. From (7), we have $p_{i_{s}}<\underline{p}$ and $d_{i_{s}}=C_{i_{s}} \underline{p} / p_{i_{s}}$, leading to

$$
R_{i_{s}}=C_{i_{s}} \underline{p}-\ell_{i_{s}}\left(C_{i_{s}} \frac{\underline{p}}{p_{i_{s}}}\right) .
$$

Consider provider $i_{s}$ unilaterally increasing its unit price $p_{i_{s}}$ to $p_{i_{s}}^{\mathrm{n}}$, with $p_{i_{s}}<p_{i_{s}}^{\mathrm{n}}<\underline{p}$ Then from (12) we have

$$
\begin{aligned}
& D(\underline{p}) \leq \sum_{i \in \mathcal{I}} C_{i} \mathbb{1}_{\underline{p} \geq p_{i}}=\sum_{i \in \mathcal{I}} C_{i} \mathbb{1}_{\underline{p} \geq p_{i}^{\mathrm{n}}} \\
& \text { and } p<\underline{p} \Rightarrow D(p)>\sum_{i \in \mathcal{I}} C_{i} \mathbb{1}_{p \geq p_{i}} \geq \sum_{i \in \mathcal{I}} C_{i} \mathbb{1}_{p \geq p_{i}^{\mathrm{n}}},
\end{aligned}
$$

which implies (again from (12), but applied to the new price profile) that the perceived price at the new Wardrop equilibrium is unchanged: $\underline{p}^{\mathrm{n}}=p$. Therefore, since $p_{i_{s}}^{\mathrm{n}}<\underline{p}$ by hypothesis, Relation (30) is still valid with new prices, and the revenue change for provider $i_{s}$ is

$$
R_{i_{s}}^{\mathrm{n}}-R_{i_{s}}=\ell_{i_{s}}\left(C_{i_{s}} \frac{\underline{p}}{p_{i_{s}}}\right)-\ell_{i_{s}}\left(C_{i_{s}} \frac{\underline{p}}{p_{i_{s}}^{\mathrm{n}}}\right)>0,
$$

due to the strict increasingness of $\ell_{i_{s}}$. This contradicts the fact that $\mathbf{p}$ is a Nash equilibrium and as a consequence,

$$
\text { at a Nash equilibrium, } \quad \mathcal{I}_{s}=\emptyset \text {. }
$$


Assume now that $\mathcal{I}_{u} \neq \emptyset$ at a Nash equilibrium price profile $\mathbf{p}$. Since we necessarily have $\mathcal{I}_{s}=\emptyset$, then from $(9), D(\underline{p})=\sum_{i \in \mathcal{I}} d_{i}<\sum_{i \in \mathcal{I}} C_{i}=D\left(p^{*}\right)$, with $p^{*}=v\left(\sum_{i \in \mathcal{I}} C_{i}\right)$. This implies that

$$
\underline{p}>p^{*}
$$

We first briefly rule out the possibility that $D(\underline{p})=0$ : if it were the case, all providers $i \in \mathcal{I}$ would have profit $-\ell_{i}(0)$. But any provider $i \in \mathcal{I}$ unilaterally changing his price to $p_{i}^{\mathrm{n}}=v\left(C_{i}\right)$ would get a total demand $C_{i}$ and obtain profit

$$
R_{i}^{\mathrm{n}}-R_{i}=C_{i} v\left(C_{i}\right)-\ell_{i}\left(C_{i}\right)+\ell_{i}(0) .
$$

Since $v\left(C_{i}\right)>v\left(\sum_{i} C_{i}\right)=p^{*}$, under Assumption A, we have $R_{i}^{\mathrm{n}}-R_{i}>C_{i}\left(p^{*}-\kappa_{i}\right) \geq 0$. Thus $R_{i}^{\mathrm{n}}>R_{i}$, a contradiction. Consequently, at a Nash equilibrium $D(\underline{p})>0$.

Now, the assumption $\mathcal{I}_{u} \neq \emptyset$ implies that there exists a provider $i_{u}$ such that

$$
d_{i_{u}}<\min \left(C_{i_{u}}, D(\underline{p})\right) .
$$

Indeed, there exists at least a provider in $\mathcal{I}_{u}$, and if that provider does not verify (33), then he gets all the demand $D(\underline{p})$, and therefore every other provider $i$ has demand $d_{i}=0<\min \left(C_{i}, D(\underline{p})\right)$ and verifies $(33)$.

Recall that every provider $i \in \mathcal{I}$ has $p_{i} \geq \underline{p}$, from (8) and $\mathcal{I}_{s}$ being empty. We now prove that provider $i_{u}$ can strictly improve its benefit by changing its price from $p_{i_{u}} \geq \underline{p}$ to $p_{i_{u}}^{\varepsilon}:=\underline{p}-\varepsilon$ for a sufficiently small $\varepsilon>0$. We distinguish two cases.

- If $C_{i_{u}} \leq D(\underline{p})$, then we easily see from (12) that the new perceived price $\underline{p}^{\varepsilon}$ verifies

$$
p_{i_{u}}^{\varepsilon}=\underline{p}-\varepsilon<\underline{p}^{\varepsilon} \leq \underline{p} .
$$

By changing its price to $\underline{p}-\varepsilon$, provider $i_{u}$ is the only provider with the lowest declared unit price, therefore from (7)-(8), its new demand $d_{i_{u}}^{\varepsilon}$ equals $C_{i_{u}} \frac{\underline{p}^{\varepsilon}}{\underline{p}-\varepsilon}$, which tends to $C_{i_{u}}$ when $\varepsilon$ tends to 0 .

- If $C_{i_{u}}>D(\underline{p})$ then for $\varepsilon$ sufficiently small (such that $D(\underline{p}-\varepsilon) \leq C_{i_{u}}$ ), provider $i_{u}$ gets all the demand, i.e., $d_{i_{u}}^{\varepsilon}=D(\underline{p}-\varepsilon)$. When $\varepsilon$ tends to 0 , that demand tends to $D(p)$ because of the continuity of the demand function.

Consequently, for a sufficiently small $\varepsilon$, the demand for provider $i_{u}$ of switching from price $p_{i_{u}}$ to price $\underline{p}-\varepsilon$ can be arbitrarily close to $y:=\min \left(C_{i_{u}}, D(\underline{p})\right)>d_{i_{u}}$, and the corresponding revenue gain can then be arbitrarily close to

$$
\begin{aligned}
\underline{p}\left(y-d_{i_{u}}\right)-\ell_{i_{u}}(y)+\ell_{i_{u}}\left(d_{i_{u}}\right. & \geq\left(\underline{p}-\kappa_{i}\right) \underbrace{\left(y-d_{i_{u}}\right)}_{>0} \\
& \geq\left(\underline{p}-p^{*}\right)\left(y-d_{i_{u}}\right) \\
& >0,
\end{aligned}
$$

where the first and second line come from $y \leq C_{i_{u}}$ and Assumption $\mathrm{A}$, and the last line stems from (32). Consequently, provider $i_{u}$ can strictly improve its net benefit by unilaterally changing its declared price, which contradicts the Nash equilibrium condition and establishes that we necessarily have

$$
\text { at a Nash equilibrium, } \quad \mathcal{I}_{u}=\emptyset .
$$


Relations (31) and (34) imply that at a Nash equilibrium, $d_{i}=C_{i}$ for all $i \in \mathcal{I}$. Then the demand relation (9) implies that $p=p^{*}$, while (7) gives $p_{i}=p$ for all $i \in \mathcal{I}$. At a Nash equilibrium, each provider $i$ necessarily declares unit price $p_{i}=p^{*}$. Step 2: $p_{i}=p^{*}, \forall i$ is a Nash equilibrium.

We now consider the price profile $\mathbf{p}$ such that $p_{i}=p^{*}, \forall i$. For that price profile, we have $d_{i}=C_{i}$ for all $i \in \mathcal{I}$, and $p=p^{*}$. First note that all providers $i \in \mathcal{I}$ get a revenue larger than $-\ell_{i}(0)$ :

$$
R_{i}+\ell_{i}(0)=p^{*} C_{i}-\left(\ell_{i}\left(C_{i}\right)-\ell_{i}(0)\right) \geq C_{i}\left(p^{*}-\kappa_{i}\right) \geq 0,
$$

where the inequalities come from Assumption A.

Let us now prove that no provider has an incentive to change his price if all the others keep their price to $p^{*}$. Without loss of generality, consider a possible move of provider 1 from $p^{*}$ to $p_{1}^{\mathrm{n}} \neq p^{*}$. We distinguish two cases.

- If $p_{1}^{\mathrm{n}}<p^{*}$, then

$$
\begin{array}{r}
D\left(p^{*}\right)=\sum_{i \in \mathcal{I}} C_{i}=\sum_{i \in \mathcal{I}} C_{i} \mathbb{1}_{\left\{p^{*} \geq p_{i}^{\mathrm{n}}\right\}} \\
\text { and } p<p^{*} \Rightarrow D(p)>\sum_{i \in \mathcal{I}} C_{i} \geq \sum_{i \in \mathcal{I}} C_{i} \mathbb{1}_{\left\{p \geq p_{i}^{\mathrm{n}}\right\}},
\end{array}
$$

which from (12) means that $\underline{p}^{\mathrm{n}}=p^{*}$. Therefore, (8) and (7) imply that $d_{1}^{\mathrm{n}}=$ $C_{1} p^{*} / p_{1}^{\mathrm{n}}$. The revenue difference for provider 1 is thus

$$
R_{1}^{\mathrm{n}}-R_{1}=\ell_{1}\left(C_{1}\right)-\ell_{1}\left(C_{1} \frac{p^{*}}{p_{1}^{\mathrm{n}}}\right) \leq 0
$$

where the last inequality comes from the nonincreasingness of $\ell_{1}$. Remark that $R_{1}^{\mathrm{n}}<R_{1}$ if $\ell_{1}$ is strictly increasing.

- If $p_{1}^{\mathrm{n}}>p^{*}$, then

$$
p \leq p^{*} \Rightarrow D(p)>\sum_{i \neq 1} C_{i} \geq \sum_{i \in \mathcal{I}} C_{i} \mathbb{1}_{p \geq p_{i}^{\mathrm{n}}}
$$

Moreover, since all providers $i \in \mathcal{I}$ have a price $p_{i}^{\mathrm{n}}=p^{*} \leq p_{1}^{\mathrm{n}}$, then

$$
D\left(p_{1}^{\mathrm{n}}\right) \leq D\left(p^{*}\right)=\sum_{i \in \mathcal{I}} \mathbb{1}_{\left\{p_{1}^{\mathrm{n}} \geq p_{i}^{\mathrm{n}}\right\}} C_{i}
$$

As a result, from (12) the new perceived price $\underline{p}^{\mathrm{n}}$ is such that

$$
p^{*}<\underline{p}^{\mathrm{n}} \leq p_{1}^{\mathrm{n}}
$$

If $p^{\mathrm{n}}<p_{1}^{\mathrm{n}}$ then $d_{1}^{\mathrm{n}}=0$ from (7), and $R_{1}^{\mathrm{n}}=-\ell_{1}(0) \leq R_{1}$ from (35).

If $\underline{\underline{p}}^{\mathrm{n}}=p_{1}^{\mathrm{n}}$ then (8) implies that $d_{1}^{\mathrm{n}} \leq C_{1}$ and $d_{i}^{\mathrm{n}}>C_{i}$ for all $i \neq 1$. Therefore(9) implies that

$$
d_{1}^{\mathrm{n}}=D\left(p_{1}^{\mathrm{n}}\right)-\sum_{i \neq 1} C_{i}
$$


and the revenue change for provider 1 is

$$
\begin{aligned}
R_{1}^{\mathrm{n}}-R_{1}= & p_{1}^{\mathrm{n}} d_{1}^{\mathrm{n}}-\ell_{1}\left(d_{1}^{\mathrm{n}}\right)-p^{*} C_{1}+\ell_{1}\left(C_{1}\right) \\
\leq & p_{1}^{\mathrm{n}}\left(D\left(p_{1}^{\mathrm{n}}\right)-\sum_{i \neq 1} C_{i}\right)-p^{*} C_{1} \\
& +\kappa_{1}\left(\sum_{i \in \mathcal{I}} C_{i}-D\left(p_{1}^{\mathrm{n}}\right)\right) \\
< & p_{1}^{\mathrm{n}} D\left(p_{1}^{\mathrm{n}}\right)-p^{*} D\left(p^{*}\right)+\kappa_{1}\left(D\left(p^{*}\right)-D\left(p_{1}^{\mathrm{n}}\right)\right) \\
= & \left(p_{1}^{\mathrm{n}}-\kappa_{1}\right) D\left(p_{1}^{\mathrm{n}}\right)-\left(p^{*}-\kappa_{1}\right) D\left(p^{*}\right) .
\end{aligned}
$$

where the second line comes from Assumption A, and the third one from $p_{1}^{\mathrm{n}}>p^{*}$ and $D\left(p^{*}\right)=\sum_{i \in \mathcal{I}} C_{i}$. Now consider the function

$$
g(y):=\left(y-\kappa_{1}\right) D(y)-\left(p^{*}-\kappa_{1}\right) D\left(p^{*}\right) .
$$

We have $g\left(p^{*}\right)=0$. Moreover, $g$ is derivable on $\left[p^{*}, p_{1}^{\mathrm{n}}\right]$, and its derivative has the same sign as $\frac{y D^{\prime}(y)}{D(y)}+\frac{1}{1-\kappa_{1} / y}$, which is nonpositive under Assumption B. Consequently, $g\left(p_{1}^{\mathrm{n}}\right) \leq g\left(p^{*}\right)$, and going back to (36) we have $R_{1}^{\mathrm{n}}<R_{1}$, concluding the proof.

\section{Can providers lie on their capacities?}

In the previous sections, we assumed that the total capacities $\left(C_{i}\right)_{i \in \mathcal{I}}$ were common knowledge of all participants. While this may not be true in reality, we may consider that providers be asked to declare their capacity level at the very beginning of the interaction, i.e., before choosing their prices, or that the used capacities have been learnt. A question that then naturally arises, since providers are still assumed to be selfish, is related to the capacity declaration strategy: is there an interest to lie on one's capacity? In this section, we answer negatively to that question, by proving that truthfulness is a dominant strategy for providers under Assumption B. As a result, even if we add a third level -a game on declared capacities- on the considered interaction -game on prices plus user choices-, there is still a unique equilibrium, that is socially efficient.

To establish that result, we assume now that each provider $i \in \mathcal{I}$ has to declare its capacity value $C_{i}$, and denote by $C_{i}^{\text {dec }}$ the value that it chooses to declare. First, remark that only the declared values $C_{i}^{\text {dec }} \leq C_{i}$ are feasible: whereas provider $i$ can easily artificially degrade its service rate, it cannot increase it above its real capacity $C_{i}$ : a false declaration aimed at increasing one's demand to get a larger benefit would be detected.

We assume that the capacity declaration occurs before the providers set their price, i.e., they commit to a certain service rate $C_{i}^{\text {dec }}$. Then from Proposition 4 , providers know that price competition will lead to a unique Nash equilibrium where all providers declare the same unit price

$$
p^{\mathrm{NE}}=v\left(\sum_{i} C_{i}^{\mathrm{dec}}\right),
$$


and each provider $i$ gets demand $C_{i}^{\text {dec }}$. Providers should therefore use that knowledge when choosing the capacity level to declare.

Focusing on the net revenue of a provider, there are two opposite effects of declaring a falsely low capacity $C_{i}^{\text {dec }}<C_{i}$ instead of the real capacity $C_{i}$ :

- since the total available capacity decreases, from (38) the unit selling price at equilibrium increases, and the managing cost decreases because the quantity sold decreases;

- on the other hand, less quantity sold means less revenue.

The next proposition gives a sufficient condition for the latter effect to overcome the former.

Proposition 5 Consider that providers can artificially lower their capacity. Under Assumption B, truthfully declaring one's real capacity is a dominant strategy for each provider.

Moreover, all providers truthfully declaring their capacities is the only Nash equilibrium of the capacity declaration game, and is a strict equilibrium.

First recall from Game Theory that a strict Nash equilibrium is a strategy profile such that each player is strictly worse off by any unilateral deviation.

Proof Without loss of generality, we prove that provider 1 strictly decreases its revenue by declaring a capacity $C_{1}^{\mathrm{U}}<C_{1}$, when each competitor $i \in \mathcal{I} \backslash\{1\}$ declares $C_{i}^{\text {dec }} \leq C_{i}$.

First remark that due to the nonincreasingness of $v$, Assumption B still holds with declared capacities and the corresponding price $p^{*}=p^{\mathrm{NE}}$. Therefore, the equilibrium of the price competition game is unique and given by Proposition 4 with those declared capacities. In other words, if we define $C_{-1}^{\mathrm{dec}}:=\sum_{i \neq 1} C_{i}^{\mathrm{dec}}$, the unit price $p^{\mathrm{NE}}$ at the price competition equilibrium is then

$$
p^{\mathrm{NE}}=v\left(C_{1}^{\mathrm{dec}}+C_{-1}^{\mathrm{dec}}\right) .
$$

Each provider $i \in \mathcal{I}$ gets demand $C_{i}^{\text {dec }}$, and gets total benefit $R_{i}=C_{i}^{\text {dec }} p^{\mathrm{NE}}-\ell_{1}\left(C_{1}^{\text {dec }}\right)$. Notice that $p^{\mathrm{NE}} \geq p^{*}$.

Now let us compare any untruthful declaration $C_{1}^{\text {dec }}=C_{1}^{\mathrm{U}}<C_{1}$, leading to provider 1 revenue $R_{1}^{\mathrm{U}}$, to the truthful declaration $C_{1}^{\mathrm{dec}}=C_{1}$, with provider 1 revenue $R_{1}^{\mathrm{T}}$. We have

$R_{1}^{\mathrm{U}}-R_{1}^{\mathrm{T}}$

$$
\begin{aligned}
& =C_{1}^{\mathrm{U}} v\left(C_{1}^{\mathrm{U}}+C_{-1}^{\mathrm{dec}}\right)-C_{1} v\left(C_{1}+C_{-1}^{\mathrm{dec}}\right)+\ell_{1}\left(C_{1}\right)-\ell_{1}\left(C_{1}^{\mathrm{U}}\right) \\
& =\left(D\left(\underline{p}^{\mathrm{U}}\right)-C_{-1}^{\mathrm{dec}}\right) \underline{p}^{\mathrm{U}}+\left(D\left(\underline{p}^{\mathrm{T}}\right)-C_{-1}^{\mathrm{dec}}\right) \underline{p}^{\mathrm{T}}+\ell_{1}\left(C_{1}\right)-\ell_{1}\left(C_{1}^{\mathrm{U}}\right),
\end{aligned}
$$

where $p^{\mathrm{U}}:=v\left(C_{1}^{\mathrm{U}}+C_{-1}^{\mathrm{dec}}\right)$, and $p^{\mathrm{T}}:=v\left(C_{1}+C_{-1}^{\mathrm{dec}}\right)$. The second equality comes from $D\left(\underline{p}^{\mathrm{U}}\right)^{-}=C_{1}^{\mathrm{U}}+C_{-1}^{\mathrm{dec}}$ and $D\left(\underline{p}^{\mathrm{T}}\right)=C_{1}+C_{-1}^{\text {dec }}$. Remark that $\underline{p}^{\mathrm{U}}>\underline{p}^{\mathrm{T}}$ since $D$ is nonincreasing.

From Assumption A, we have

$R_{1}^{\mathrm{U}}-R_{1}^{\mathrm{T}}$

$$
\begin{aligned}
& \left.\unlhd D\left(\underline{p}^{\mathrm{U}}\right)-C_{-1}^{\mathrm{dec}}\right) \underline{p}^{\mathrm{U}}+\left(D\left(\underline{p}^{\mathrm{T}}\right)-C_{-1}^{\mathrm{dec}}\right) \underline{p}^{\mathrm{T}}+\kappa_{1}\left(C_{1}-C_{1}^{\mathrm{U}}\right) \\
& =\underbrace{-\left(\underline{p}^{\mathrm{U}}-\underline{p}^{\mathrm{T}}\right)}_{<0} C_{-1}^{\mathrm{dec}}+\underline{p}^{\mathrm{U}} D\left(\underline{p}^{\mathrm{U}}\right)-\underline{p}^{\mathrm{T}} D \underline{p}^{\mathrm{T}}+\kappa_{1} \underbrace{\left(C_{1}-C_{1}^{\mathrm{U}}\right)}_{=D\left(\underline{p}^{\mathrm{T}}\right)-D\left(\underline{p}^{\mathrm{U}}\right)}, \\
& \unlhd\left(\underline{p}^{\mathrm{U}}-\kappa_{1}\right) D\left(\underline{p}^{\mathrm{U}}\right)-\left(\underline{p}^{\mathrm{T}}-\kappa_{1}\right) D\left(\underline{p}^{\mathrm{T}}\right) .
\end{aligned}
$$


The last line, taken as a function of $\underline{p}^{\mathrm{U}}$, is of the same form as in (37), and is therefore nonincreasing for $\underline{p}^{\mathrm{U}} \geq \underline{p}^{\mathrm{T}}$ under Assumption B. Since it is null at $\underline{p}^{\mathrm{T}}$, then $\underline{p}^{\mathrm{U}}>\underline{p}^{\mathrm{T}}$ yields $R_{1}^{\mathrm{U}} \leq R_{1}^{\mathrm{T}}$, which proves that truthful declaration is a dominant strategy. As a result, all providers being truthful is a Nash equilibrium of the price declaration game.

Remark that as soon as one competitor declares a non-zero capacity, then $C_{-1}^{\text {dec }}>0$, and the inequality in (40) is strict. Therefore, the (truthful) Nash equilibrium is a strict equilibrium, and the only possible other equilibrium would consist in all providers declaring a null capacity, i.e., $C_{i}^{\text {dec }}=0$ for all $i \in \mathcal{I}$. We now exclude that possibility.

Consider provider 1 , and assume all its competitors declare a null capacity, i.e., $C_{-1}^{\text {dec }}=0$. By declaring a null capacity $C_{1}^{\text {dec }}=0$, provider 1 would get a total revenue $R_{1}^{\mathrm{U}}=-\ell_{1}(0)$. However declaring its true capacity $C_{1}$ and setting its price to $v\left(C_{1}\right)$ would yield a revenue $R_{1}^{\mathrm{T}}=C_{1} v\left(C_{1}\right)-\ell_{1}\left(C_{1}\right)$. Under Assumption A, the revenue gain is therefore

$$
R_{1}^{\mathrm{T}}-R_{1}^{\mathrm{U}} \geq C_{1}\left(v\left(C_{1}\right)-\kappa_{1}\right)>0,
$$

where the strict inequality comes from the strict decreasingness of $v$. As a result, provider 1 has an interest to deviate from the situation where providers declare zero capacity, which rules out that situation for being a Nash equilibrium, and concludes the proof.

As a consequence of Proposition 5, even if providers have the possibility to artificially reduce their service capacity before fixing their prices, the final outcome of the competition game still corresponds to the socially efficient situation pointed out in Proposition 2.

\section{Conclusion}

This paper provides an analysis of a pricing game among competitive telecommunication service providers with potentially different but fixed capacities. According to the price profile, we have been able to define and characterize the demand repartition for selfish infinitesimal users, applying Wardrop's principle. Using the knowledge of what this repartition would be, providers can play with their price in order to maximize their revenue. We have proved the existence of a unique Nash equilibrium for that game, where all providers set the same price, for which demand exactly meets the sum of capacities. We have also established that providers have no incentive to artificially create some congestion by declaring a falsely low capacity. It turns out that those interactions among selfish agents (providers and customers) lead to an outcome that maximizes social welfare, i.e., available network resources are optimally used.

As extensions of this work, we would like to investigate the viability of (or to define rules to make viable) scenarii that might be more specific to wireless. For instance when a provider is a virtual operator leasing capacity to a competitor owning a license. Other scenarii of interest would regard cognitive networks, i.e., the case when unused capacity can be used by secondary users. In general, considering a capacity expansion game is also an interesting issue. Indeed, capacity can be an important parameter providers can play with, at the same time as prices: what would the resulting equilibrium be?

Acknowledgements The authors would like to acknowledge the support of Euro-NF Network of Excellence, and the French National Research Agency through the CAPTURES project and the WINEM project for the second author. 


\section{References}

1. Aashtiani, H.Z., Magnanti, T.L.: Equilibria on a congested transportation network. SIAM Journal of Algebraic and Discrete Methods 2, 213-226 (1981)

2. Acemoglu, D., Ozdaglar, A.: Competition and efficiency in congested markets. Mathematics of Operations Research (2006)

3. Acemoglu, D., Ozdaglar, A.: Price competition in communication networks. In: Proc. of IEEE INFOCOM (2006)

4. Altman, E., Boulogne, T., El-Azouzi, Jiménez, T., Wynter, L.: A survey on networking games in telecommunications. Computers and Operations Research (2005). Available on the journal's home page as well as in the authors' home pages

5. Correa, J.R., Figueroa, N., Stier-Moses, N.: Pricing with markups in industries with increasing marginal costs. Columbia Working Paper \#DRO-2008-14 (2008)

6. Courcoubetis, C., Weber, R.: Pricing Communication Networks-Economics, Technology and Modelling. Wiley (2003)

7. DaSilva, L.: Pricing of QoS-Enabled Networks: A Survey. IEEE Communications Surveys \& Tutorials 3(2) (2000)

8. El-Azouzi, R., Altman, E., Wynter, L.: Telecommunications network equilibrium with price and quality-of-service characteristics. In: Proc. of 18th International Teletraffic Congress. Berlin, Germany (2003)

9. Felegyhazi, M., Hubaux, J.: Wireless operators in a shared spectrum. In: Proc. of IEEE INFOCOM (2006)

10. Fudenberg, D., Tirole, J.: Game Theory. MIT Press, Cambridge, Massachusetts (1991)

11. Gibbens, R., Mason, R., Steinberg, R.: Internet service classes under competition. IEEE Journal on Selected Areas in Communications 18(12), 2490-2498 (2000)

12. Hayrapetyan, A., Tardos, E., Wexler, T.: A network pricing game for selfish traffic. In: Proc. of IEEE PODC (2006)

13. Ileri, O., Samardzija, D., Sizer, T., Mandayam, N.: Demand responsive pricing and competitive spectrum allocation via a spectrum server. In: Proc. of IEEE DySpan 2005 (2006)

14. Le Cadre, H., Bouthou, M., Tuffin, B.: Modeling consumers' preference to price bundle offers in telecommunication industry game when competition among operators. Netnomics 10(9), 171-208 (2009)

15. Liu, Z., Wynter, L., Xia, C.: Pricing information services in a competitive market: avoiding price wars. Tech. rep., INRIA (2002)

16. Maillé, P., Tuffin, B.: Analysis of price competition in a slotted resource allocation game. In: Proc. of IEEE INFOCOM. Phoenix, AZ, USA (2008)

17. Manshaei, M.H., Freudiger, J., Félegyházi, M., Marbach, P., Hubaux, J.P.: On wireless social community networks. In: Proc. of IEEE INFOCOM. Phoenix, AZ, USA (2008)

18. Reichl, P., Stiller, B.: Nil nove sub sole: Why internet charging schemes look like as they do. In: Proceedings of the 4th Berlin Internet Economic Workshop (2001)

19. Stiller, B., Reichl, P., Leinen, S.: Pricing and Cost Recovery for Internet Services: Practical Review, Classification, and Application of Relevant Models. Netnomics 2(1) (2000)

20. Tuffin, B.: Charging the Internet without bandwidth reservation: an overview and bibliography of mathematical approaches. Journal of Information Science and Engineering 19(5), 765-786 (2003)

21. Vives, X.: Oligopoly Pricing, Old Ideas and New Tools. The MIT Press (2001)

22. Wardrop, J.: Some theoretical aspects of road traffic research. proceedings of the Institute of Civil Engineers 1, 325-378 (1957) 